\title{
Continuous Semiring-Semimodule Pairs and Mixed Algebraic Systems*
}

\author{
Zoltán Ésik ${ }^{a}$ and Werner Kuich ${ }^{b}$
}

\begin{abstract}
We associate with every commutative continuous semiring $S$ and alphabet $\Sigma$ a category whose objects are all sets and a morphism $X \rightarrow Y$ is determined by a function from $X$ into the semiring of formal series $S\left\langle\left\langle(Y \biguplus \Sigma)^{*}\right\rangle\right.$ of finite words over $Y \biguplus \Sigma$, an $X \times Y$-matrix over $S\left\langle(Y \biguplus \Sigma)^{*}\right\rangle$, and a function from $X$ into the continuous $S\left\langle(Y \biguplus \Sigma)^{*}\right\rangle$-semimodule $S\left\langle\left\langle(Y \biguplus \Sigma)^{\omega}\right\rangle\right.$ of series of $\omega$ words over $Y \biguplus \Sigma$. When $S$ is also an $\omega$-semiring (equipped with an infinite product operation), then we define a fixed point operation over our category and show that it satisfies all identities of iteration categories. We then use this fixed point operation to give semantics to recursion schemes defining series of finite and infinite words. In the particular case when the semiring is the Boolean semiring, we obtain the context-free languages of finite and $\omega$-words.
\end{abstract}

\section{Introduction}

Suppose that $S$ is a continuous semiring and $\Sigma$ and $X$ are sets. Let $X^{*}$ and $X^{\omega}$ respectively denote the sets of all finite and $\omega$-words over $X$. We can form the continuous semiring $S\left\langle\left\langle X^{*}\right\rangle\right.$ of series over $X^{*}$ with coefficients in $S$ and the continuous $S\left\langle\left\langle X^{*}\right\rangle\right.$-semimodule $S\left\langle\left\langle X^{\omega}\right\rangle\right\rangle$ of series over $X^{\omega}$ with coefficients in $S$. If $S$ is equipped with an infinite product operation $S^{\omega} \rightarrow S, s_{1} s_{2} \cdots \mapsto \prod_{n \geq 1} s_{n}$, satisfying certain axioms including a sort of continuity described in the sequel, then we can also define an infinite product operation $\left(S\left\langle\left\langle X^{*}\right\rangle\right)^{\omega} \rightarrow S\left\langle\left\langle X^{\omega}\right\rangle\right.\right.$. In our first result, we show that the construction of the "continuous $\omega$-semiring-semimodule pair' $\left(S\left\langle\left\langle X^{*}\right\rangle, S\left\langle\left\langle X^{\omega}\right\rangle\right)\right.\right.$ enjoys a universal property, cf. Theorem 5.1.

In the second part of the paper we use the above universality result to give an algebraic treatment of recursion schemes defining series of finite and infinite words over $\Sigma$. To this end, we will restrict ourselves to commutative continuous

\footnotetext{
* The first author received support from NKFI grant no. ANN 110883. The second author was partially supported by Austrian Science Fund (FWF): grant no. I1661 -N25.

${ }^{a}$ Dept. of Foundations of Computer Science, University of Szeged, Szeged, Hungary

${ }^{b}$ Inst. of Discrete Mathematics and Geometry, Technical University of Vienna, Vienna, Austria E-mail: kuich@tuwien.ac.at
} 
$\omega$-semirings $S$. Suppose that

$$
x_{i}=p_{i} i \in I(R)
$$

is a finite or infinite recursion scheme (or system of fixed point equations), where each $p_{i}$ is a series in $S\left\langle(X \uplus \Sigma)^{*}\right\rangle$. Then we associate with $R$ in a natural way a function

$$
F_{R}: S\left\langle\left\langle\Sigma^{*}\right\rangle\right\rangle \times S\left\langle\left\langle S^{\omega}\right\rangle\right\rangle \rightarrow S\left\langle\left\langle\Sigma^{*}\right\rangle \times S\left\langle\left\langle S^{\omega}\right\rangle\right\rangle\right.
$$

and define the semantics of $R$ as a 'canonical' fixed point of $F_{R}$.

In order to facilitate the construction of the canonical fixed point, we introduce a category whose objects are sets (of recursion variables) and a morphism $f: X \rightarrow Y$ has three components:

- a function $\left.f_{0}: X \rightarrow S\left\langle(Y \uplus \Sigma)^{*}\right\rangle\right\rangle$,

- a matrix $f_{M} \in\left(S\left\langle(Y \uplus \Sigma)^{*}\right\rangle\right)^{X \times Y}$,

- a function $f_{\omega}: X \rightarrow S\left\langle(Y \uplus \Sigma)^{\omega}\right\rangle$.

We define composition and the identity morphisms in a natural way to obtain a category $\operatorname{Ser}_{S, \Sigma}^{\omega}$. By taking the first components of morphisms, this category can be projected onto the category $\mathbf{S e r}_{S, \Sigma}$ having sets as objects and functions $X \rightarrow S\left\langle\left\langle(Y \uplus \Sigma)^{*}\right\rangle\right\rangle$ as morphisms $X \rightarrow Y$. It will be clear from the definition that the coproduct $X_{1} \oplus \cdots \oplus X_{n}$ of any finite sequence $X_{1}, \ldots, X_{n}$ of objects exists in $\operatorname{Ser}_{S, \Sigma}^{\omega}$, in fact it will be given by disjoint union. (Actually all coproducts will exist, but this fact is not important for the paper.)

Next we define a dagger operation mapping a morphism $f: X \rightarrow X \oplus Y$ to a morphism $f^{\dagger}: X \rightarrow Y$. We prove that for any $f: X \rightarrow X \oplus Y$, the morphism $f^{\dagger}: X \rightarrow Y$ is a solution of the fixed point equation

$$
\xi=f \circ\left\langle\xi, \mathbf{i d}_{Y}\right\rangle
$$

(Here, $\langle-,-\rangle$ denotes the source pairing operation determined by the coproduct structure.) Intuitively, $f$ represents a system of fixed point equations (recursion scheme) in the variables $X$ and parameters $Y$, and $f^{\dagger}$ is its canonical solution. In particular, the function $F_{R}$ associated with a system of fixed point equations $(R)$ can be seen as a morphism $f: X \rightarrow X$ in $\operatorname{Ser}_{S, \Sigma}^{\omega}$, and the canonical fixed point of $F_{R}$ can be derived from $f^{\dagger}: X \rightarrow \varnothing$. Indeed the three components of $f^{\dagger}$ are a function $X \rightarrow S\left\langle\left\langle\Sigma^{*}\right\rangle\right\rangle$, the empty $X \times \varnothing$-matrix over $S\left\langle\left\langle\Sigma^{*}\right\rangle\right\rangle$, and a function $X \rightarrow S\left\langle\left\langle\Sigma^{\omega}\right\rangle\right\rangle$. The first and third components form the canonical fixed point of $F_{R}$. Our approach generalizes the construction of context-free languages of finite and $\omega$-words [10].

Categories with finite coproducts (or dually, products) equipped with a parametric fixed point operation have been studied since the late 1960's. A class of structures, called iteration theories, or iteration categories, have been identified. It has been shown that most (if not all) of the major fixed point structures used in computer science give rise to iteration categories. As a main technical contribution, 
we prove that for each commutative continuous semiring $S$ and alphabet $\Sigma$, $\operatorname{Ser}_{S, \Sigma}^{\omega}$ is also an iteration category, cf. Theorem 7.1. A few consequences of this fact are discussed in the conclusion.

\section{Continuous semirings}

Recall that a commutative monoid $(V,+, 0)$ is continuous (cf. Section 2.2 of [6]) if it is equipped with a a partial order $\leq$ such that the supremum of any chain (or equivalently, directed set [12]) exists and 0 is the least element. Moreover, the sum operation + is continuous:

$$
x+\sup Y=\sup (x+Y)
$$

for all nonempty chains or nonempty directed sets $Y \subseteq V$, where $x+Y=\{x+y$ : $y \in Y\}$. (Actually this also holds when the set is empty.) It follows that the sum operation is monotonic: if $x \leq y$ in $V$, then $x+z \leq y+z$ for all $z \in V$.

Suppose that $V$ is a continuous commutative monoid and $x_{i} \in V$ for all $i \in I$. We define $\sum_{i \in I} x_{i}$ as the supremum of all finite sums $x_{i_{1}}+\ldots+x_{i_{n}}$ where $i_{1}, \ldots, i_{n}$ are pairwise different elements of $I$. It is well-known that this summation operation is completely associative and commutative:

$$
\sum_{i \in I} x_{i}=\sum_{j \in J} \sum_{i \in I_{j}} x_{i}
$$

whenever $I$ is the disjoint union of the sets $I_{j}, j \in J$ and $x_{i} \in V$ for all $i \in I$. Moreover,

$$
\sum_{i \in I} x_{i}=\sum_{i \in I} x_{\pi(i)}
$$

whenever $\pi$ is a permutation $I \rightarrow I$ and $x_{i} \in V$ for all $i \in I$.

Continuous commutative monoids are closed under several constructions including direct product. Suppose that $V_{i}$ is a continuous commutative monoid for all $i \in I$. Then $V=\prod_{i \in I} V_{i}$, equipped with the pointwise sum operation and pointwise ordering, is also a continuous commutative monoid. It follows that the summation operation in the product $V$ is the pointwise summation. In particular, if $V$ is a continuous commutative monoid, then so is $V^{I}$ for any set $I$.

Suppose now that $S=(S,+, \cdot, 0,1)$ is a semiring $[8,11]$. We say that $S$ is a continuous semiring (cf. Section 2.2 of $[6]$ ) if $(S,+, 0)$ is a continuous commutative monoid equipped with a partial order $\leq^{1}$ and the product operation is continuous (hence, also monotonic), i.e., it preserves the supremum of nonempty chains (or nonempty directed subsets) in either argument:

$$
\begin{aligned}
& (\sup X) y=\sup (X y) \\
& y(\sup X)=\sup (y X),
\end{aligned}
$$

\footnotetext{
${ }^{1}$ Unlike at some other places, we do not require that $x \leq y$ iff there is some $z$ with $x+z=y$.
} 
for all nonempty chains (or directed sets) $X \subseteq S$, where $X y=\{x y: x \in X\}$ and $y X$ is defined in the same way. It follows that product distributes over all sums:

$$
\begin{aligned}
\left(\sum_{i \in I} x_{i}\right) y & =\sum_{i \in I} x_{i} y \\
y\left(\sum_{i \in I} x_{i}\right) & =\sum_{i \in I} y x_{i}
\end{aligned}
$$

whenever $x_{i} \in S$ for all $i \in I$.

Suppose that $S$ is a continuous semiring and $I$ is a set. Then the matrix semiring $S^{I \times I}$, equipped with the pointwise sum operation, the usual matrix product operation and the pointwise ordering is also a continuous semiring. The matrix product is meaningful since the sum of any $I$-indexed family of elements of $S$ exists.

Continuous semirings are also closed under the formation of power series semirings. Suppose that $S$ is a continuous semiring and $X$ is a set. As usual, let $S\left\langle\left\langle X^{*}\right\rangle\right.$ denote the semiring of all power series $s=\sum_{u \in X^{*}}\langle s, u\rangle u$ over $X$ with coefficients in $S$. Each series $s$ may be viewed as a function $X^{*} \rightarrow S$ mapping a word $u \in X^{*}$ to $\langle s, u\rangle$. Equipped with the pointwise order relation $s \leq s^{\prime}$ iff $\langle s, u\rangle \leq\left\langle s^{\prime}, u\right\rangle$ for all $u \in X^{*}, S\left\langle\left\langle X^{*}\right\rangle\right.$ is a continuous semiring. The sum of any family of series is the pointwise sum.

Theorem 2.1. Suppose that $S$ is a continuous semiring. Then for each set $X$, the continuous semiring $S\left\langle\left\langle X^{*}\right\rangle\right.$ has the following universal property. Given a continuous semiring $S^{\prime}$, a continuous semiring morphism $h_{S}: S \rightarrow S^{\prime}$ and any function $h_{X}: X \rightarrow S^{\prime}$ such that the elements of $h_{S}(S)$ commute with the elements of $S^{\prime}$, there is a unique continuous semiring morphism $h^{\sharp}: S\left\langle\left\langle X^{*}\right\rangle \rightarrow S^{\prime}\right.$ extending $h_{S}$ and $h_{X}$.

Proof. We provide an outline of the proof. For details, see [7].

First we extend $h_{X}$ to a monoid morphism $X^{*} \rightarrow S^{\prime}$, denoted just $h$. Then, for a series $s \in S\left\langle\left\langle X^{*}\right\rangle\right\rangle$, we define $h^{\sharp}(s)=\sum_{u \in X^{*}} h_{S}(\langle s, u\rangle) h(u)$. It is clear that $h^{\sharp}$ extends $h_{S}$ and $h_{X}$ and preserves the constants 0 and 1 . Then we prove that $h^{\sharp}$ is continuous and preserves the binary sum operation. It then follows that $h^{\sharp}$ preserves all finite and infinite sums. Last, we prove that $h^{\sharp}$ preserves the product operation. Since the definition of $h^{\sharp}$ was forced, it is unique.

\section{Continuous semiring-semimodule pairs}

Suppose now that $S$ is a semiring and $V$ is a commutative monoid as above. We call $V$ a (left) $S$-semimodule [9] if there is an action $S \times V \rightarrow S$ subject to the usual 
associativity, distributivity and unitary conditions:

$$
\begin{aligned}
\left(s_{1} s_{2}\right) v & =s_{1}\left(s_{2} v\right) \\
\left(s_{1}+s_{2}\right) v & =s_{1} v+s_{2} v \\
s\left(v_{1}+v_{2}\right) & =s v_{1}+s v_{2} \\
1 v & =v \\
0 v & =0 \\
s 0 & =0
\end{aligned}
$$

for all $s, s_{1}, s_{2} \in S$ and $v, v_{1}, v_{2} \in V$. When $V$ is an $S$-semimodule, we also say that $(S, V)$ is a semiring-semimodule pair.

We call a semiring-semimodule pair $(S, V)$ continuous if $S$ is a continuous semiring and $V$ is a continuous commutative monoid such that the action is continuous in either argument:

$$
\begin{aligned}
& (\sup X) v=\sup (X v) \\
& x(\sup Y)=\sup (x Y)
\end{aligned}
$$

for all $x \in S, y \in V$ and nonempty chains (or nonempty directed sets) $X \subseteq S$ and $Y \subseteq V$. Of course, $X v=\{s v: s \in X\}$ and $x Y=\{x w: w \in Y\}$. It follows that action distributes over summation on either side:

$$
\begin{aligned}
& \left(\sum_{i \in I} x_{i}\right) v=\sum_{i \in I} x_{i} v \\
& x\left(\sum_{i \in I} v_{i}\right)=\sum_{i \in I} x v_{i}
\end{aligned}
$$

for all $x, x_{i} \in S, v, v_{i} \in V, i \in I$, where $I$ is any index set.

Moreover, we call a continuous semiring-semimodule pair $(S, V)$ a continuous $\omega$-semiring-semimodule pair if it is equipped with an infinite product operation $\prod_{n>1} x_{n}$ mapping an $\omega$-sequence (or $\omega$-word) $x_{1} x_{2} \cdots \in S^{\omega}$ to $\prod_{n \geq 1} x_{n} \in V$. The infinite product is subject to the following axioms:

Ax1

$$
x \prod_{n \geq 1} x_{n}=\prod_{n \geq 1} y_{n}
$$

where $y_{1}=x$ and $y_{n+1}=x_{n}$ for all $n \geq 1$.

Ax2

$$
\prod_{n \geq 1} x_{n}=\prod_{n \geq 1} x_{i_{n}} \cdots x_{i_{n+1}-1}
$$

where the sequence $i_{1}=1 \leq i_{2} \leq \cdots$ increases without a bound and the product of an empty family is 1 .

Ax3

$$
\prod_{n \geq 1}\left(x_{n}+y_{n}\right)=\sum_{z_{n}=x_{n}} \prod_{z_{n}=y_{n}} z_{n \geq 1}
$$


Ax 4

$$
\prod_{n \geq 1} \sup X_{n}=\sup _{x_{n} \in X_{n}} \prod_{n \geq 1} x_{n}
$$

where for each $n, X_{n} \subseteq S$ is a nonempty chain (or a nonempty directed set).

It follows that

$$
\prod_{n \geq 1} \sum_{i_{n} \in I_{n}} x_{i_{n}}=\sum_{i_{1} \in I_{1}, i_{2} \in I_{2}, \ldots} \prod_{n \geq 1} x_{i_{n}}
$$

where $\left\{x_{i_{n}}: i_{n} \in I_{n}\right\}$ is a family of elements of $S$ for all $n \geq 1$. Indeed,

$$
\begin{aligned}
\prod_{n \geq 1} \sum_{i_{n} \in I_{n}} x_{i_{n}} & =\prod_{n \geq 1} \sup _{F_{n} \subseteq I_{n}}\left\{\sum_{i_{n} \in F_{n}} x_{i_{n}}\right\} \\
& =\sup _{F_{n} \subseteq I_{n}} \prod_{n \geq 1}\left\{\sum_{i_{n} \in F_{n}} x_{i_{n}}\right\} \\
& =\sup _{F_{n} \subseteq I_{n}} \sum_{i_{n} \in F_{n}} \prod_{n \geq 1} x_{i_{n}} \\
& =\sup _{F \subseteq I_{1} \times I_{2} \times \ldots} \sum_{\left(i_{1}, i_{2}, \ldots\right) \in F} \prod_{n \geq 1} x_{i_{n}} \\
& =\sum_{\left(i_{1}, i_{2}, \ldots\right) \in I_{1} \times I_{2} \times \ldots} \prod_{n \geq 1} x_{i_{n}} .
\end{aligned}
$$

In particular, note that $\prod_{n \geq 1} x_{n}=0$ whenever there is some $m$ such that $x_{m}$ is 0. (This also follows from Ax1.) Moreover, infinite product is monotonic: if $x_{n} \leq y_{n}$ in $S$ for all $n \geq 1$, then $\prod_{n \geq 1} x_{n} \leq \prod_{n \geq 1} y_{n}$.

Suppose that $S$ is a continuous semiring. Then we define a star operation $S \rightarrow S$ as usual: $s^{*}=\sum_{n \geq 0} s^{n}$ for all $s \in S$. It is known that $s^{*}$ is the least solution of the fixed point equation $x=s x+1$ (and also of $x=x s+1$ ) over $S$. And if $(S, V)$ is a continuous $\omega$-semiring-semimodule pair, we define an omega operation $S^{\omega} \rightarrow V$ by $s^{\omega}=\prod_{n \geq 1} s$ for all $s \in S$. It is known that for each $s \in S, s^{\omega}$ is a solution of the equation $v=s v$ over $V$.

Complete equational and quasi-equational axiomatization of the equational properties of the star operation in continuous semirings has been given in [2]. Among the identities satisfied by continuous semirings are the sum star and product star identities $[3,1]$ :

$$
\begin{aligned}
(x+y)^{*} & =\left(x^{*} y\right)^{*} x^{*} \\
(x y)^{*} & =1+x(y x)^{*} y
\end{aligned}
$$

Also $0^{*}=1$ and $1^{*}=1^{* *}$ hold.

When $(S, V)$ is a continuous $\omega$-semiring-semimodule pair, the omega operation satisfies the sum omega and product omega identities [1]:

$$
\begin{aligned}
(x+y)^{\omega} & =\left(x^{*} y\right)^{\omega}+\left(x^{*} y\right)^{*} x^{\omega} \\
(x y)^{\omega} & =x(y x)^{\omega}
\end{aligned}
$$

for all $x, y \in S$. Also $0^{\omega}=0$. 


\section{Matrices and series}

\subsection{Matrices}

Suppose that $(S, V)$ is a continuous $\omega$-semiring-semimodule pair and $I$ is a set. Then, as mentioned above, $S^{I \times I}$ is a continuous semiring, and $V^{I}$ is a continuous commutative monoid. There is a natural left action of $S^{I \times I}$ on $V^{I}$ defined similarly to matrix multiplication:

$$
(M N)_{i}=\sum_{j \in I} M_{i, j} N_{j}, i \in I
$$

for all $M \in S^{I \times I}$ and $N \in S^{I}$. Moreover, we may define an infinite product operation by

$$
\left(\prod_{n \geq 1} M_{n}\right)_{i}=\sum_{i=i_{1}, i_{2}, \ldots} \prod_{n \geq 1}\left(M_{n}\right)_{i_{n}, i_{n+1}}, i \in I
$$

Proposition 4.1. Suppose that $(S, V)$ is a continuous $\omega$-semiring-semimodule pair and $I$ is a set. Then, equipped with the pointwise orderings, $\left(S^{I \times I}, V^{I}\right)$ is also a continuous $\omega$-semiring-semimodule pair.

Proof. The fact that $S^{I \times I}$ is a continuous semiring is proved in [7]. It is clear that $V^{I}$ is a continuous commutative monoid and that equipped with the action, $\left(S^{I \times I}, V^{I}\right)$ is a semiring-semimodule pair. The action is continuous in either argument, so that $\left(S^{I \times I}, V^{I}\right)$ is also a continuous semiring-semimodule pair. This can be proved by an argument similar to that used in [7] to establish that product in $S^{I \times I}$ is continuous in either argument.

In order to conclude, we still need to prove that the infinite product over matrices satisfies the axioms Ax1-Ax4. Let $M_{n} \in S^{I \times I}$ for all $n \geq 1$, and define $M^{\prime}=M_{1}$ and $M_{n}^{\prime}=M_{n+1}$ for all $n \geq 1$. Then for every $i \in I$, the $i$ th component of $\prod_{n \geq 1} M_{n}^{\prime}$ is

$$
\sum_{j \in I}\left(M_{1}\right)_{i, j} \sum_{j=j_{1}, j_{2}, \ldots \in I}\left(\prod_{n \geq 1}\left(M_{n+1}\right)_{j_{n}, j_{n+1}}\right)=\sum_{i=i_{1}, i_{2}, \ldots \in I} \prod_{n \geq 1}\left(M_{n}\right)_{i_{n}, i_{n+1}}
$$

which is the $i$ th component of $\prod_{n \geq 1} M_{n}$. Hence, Ax1 holds.

Suppose now that the sequence $1=k_{1} \leq k_{2} \leq \cdots$ increases without a bound and define $M_{n}^{\prime}=M_{i_{n}} \cdots M_{i_{n+1}-1}$ for all $n \geq 1$. Then for each $i \in I$, the $j$ th component of $\prod_{n \geq 1} M_{n}^{\prime}$ is

$$
\begin{aligned}
& \sum_{j=j_{1}, j_{2}, \ldots \in I} \prod_{n \geq 1}\left(M^{\prime}\right)_{j_{n}, j_{n+1}} \\
= & \sum_{j=j_{1}, j_{2}, \ldots \in I} \prod_{n \geq 1} \sum_{j_{n}=\ell_{1}, \ell_{2}, \ldots, \ell_{i_{n+1}-i_{n}}=j_{n+1}}\left(M_{i_{n}}\right)_{\ell_{1}, \ell_{2}} \cdots\left(M_{i_{n+1}-1}\right) \ell_{\ell_{n+1}-i_{n}-1}, \ell_{i_{n+1}-i_{n}} \\
= & \sum_{j=j_{1}, j_{2}, \ldots} \prod_{n \geq 1}\left(M_{j}\right)_{j_{n}, j_{n+1}},
\end{aligned}
$$

proving Ax2. 
In order to prove that Ax3 holds, let $M_{n}, M_{n}^{\prime} \in S^{I \times I}$ for all $n \geq 1$. Then for every $i \in I$, the $i$ th component of $\prod_{n \geq 1}\left(M_{n}+M_{n}^{\prime}\right)$ is

$$
\begin{aligned}
\sum_{i=i_{1}, i_{2}, \ldots} \prod_{n \geq 1}\left(M_{n}+M_{n}^{\prime}\right)_{i_{n}, i_{n+1}} & =\sum_{P_{n}=M_{n}} \sum_{\text {or }} \sum_{P_{n}=M_{n}^{\prime}} \sum_{i=i_{1}, i_{2}, \ldots} \prod_{n \geq 1} P_{i_{n}, i_{n+1}} \\
& =\left(\sum_{P_{n}=M_{n}} \sum_{\text {or }} P_{n}=M_{n}^{\prime} \prod_{n \geq 1} P_{n}\right)_{i},
\end{aligned}
$$

i.e., the $i$ th component $\sum_{P_{n}=M_{n}}$ or $P_{n}=M_{n}^{\prime} \prod_{n \geq 1} P_{n}$. This proves Ax3.

Suppose now that for each $n \geq 1, \mathcal{M}_{n}$ is a nonempty chain w.r.t. the pointwise ordering of matrices in $S^{I \times I}$. We want to prove that

$$
\prod_{n \geq 1} \sup \mathcal{M}_{n}=\sup _{M_{n} \in \mathcal{M}_{n}} \prod_{n \geq 1} M_{n}
$$

Let $i \in I$ be fixed. Then the $i$ th component of $\prod_{n \geq 1} \sup \mathcal{M}_{n}$ is

$$
\begin{aligned}
\sum_{i=i_{1}, i_{2}, \ldots} \prod_{n \geq 1}(\sup \mathcal{M})_{i_{n}, i_{n+1}} & =\sum_{i=i_{1}, i_{2}, \ldots} \sup _{M_{n} \in \mathcal{M}_{n}} \prod_{n \geq 1}\left(M_{n}\right)_{i_{n}, i_{n+1}} \\
& =\sup _{M_{n} \in \mathcal{M}_{n}} \sum_{i=i_{1}, i_{2}, \ldots} \prod_{n \geq 1}\left(M_{n}\right)_{i_{n}, i_{n+1}} \\
& =\sup _{M_{n} \in \mathcal{M}_{n}}\left(\prod_{n \geq 1} M_{n}\right)_{i} .
\end{aligned}
$$

Here, we used the fact, proved in [7], that summation is continuous. It follows that (2) holds.

Hence, if $(S, V)$ is a continuous $\omega$-semiring-semimodule pair, then $\left(S^{I \times I}, V^{I}\right)$ comes with a star operation and an omega operation.

\subsection{Series}

We may also construct continuous $\omega$-semiring-semimodule pairs of power series. To this end, we assume that $S$ is a continuous $\omega$-semiring equipped with an infinite product $S^{\omega} \rightarrow S$ subject to axioms similar to those defining continuous $\omega$-semiringsemimodule pairs. This amounts to requiring that, equipped with left multiplication as the action and the infinite product, $(S, S)$ is a continuous $\omega$-semiring-semimodule pair.

Let $X$ be any set. We already know that $S\left\langle\left\langle X^{*}\right\rangle\right.$ is a continuous semiring. In a similar way, $S\left\langle\left\langle X^{\omega}\right\rangle\right.$, equipped with the pointwise sum operation and pointwise ordering, is a continuous commutative monoid, and the action of $S\left\langle\left\langle X^{*}\right\rangle\right.$ on $S\left\langle\left\langle X^{\omega}\right\rangle\right\rangle$ defined by

$$
s r=\sum_{w \in A^{\omega}} \sum_{w=u v}\langle s, u\rangle\langle r, v\rangle u v
$$

turns $S\left\langle\left\langle X^{\omega}\right\rangle\right\rangle$ into an $S\left\langle\left\langle X^{*}\right\rangle\right\rangle$-semimodule. Moreover, the action is continuous in either argument and it is easy to check that Ax1-Ax4 hold. 
Let $s_{n} \in S\left\langle\left\langle X^{*}\right\rangle\right.$ for all $n \geq 1$. We define $r=\prod_{n \geq 1} s_{n} \in S\left\langle\left\langle X^{\omega}\right\rangle\right\rangle$ as follows. Given $v \in X^{\omega}$, we define

$$
\langle r, v\rangle=\sum_{v=v_{1} v_{2} \ldots} \prod_{n \geq 1}\left\langle s_{n}, v_{n}\right\rangle
$$

Proposition 4.2. Let $S$ be a continuous $\omega$-semiring and $X$ be a set. Then $\left(S\left\langle\left\langle X^{*}\right\rangle, S\left\langle\left\langle X^{\omega}\right\rangle\right)\right.\right.$ is a continuous $\omega$-semiring-semimodule pair.

Proof. First we establish Ax1. Let $s \in S\left\langle\left\langle X^{*}\right\rangle\right\rangle$ and $s_{n} \in S\left\langle\left\langle X^{*}\right\rangle\right.$ for all $n \geq 1$. Then, for all $w \in X^{\omega}$,

$$
\begin{aligned}
\left\langle s \prod_{n \geq 1} s_{n}, w\right\rangle & =\sum_{w=u v}\langle s, u\rangle\left\langle\prod_{n \geq 1} s_{n}, v\right\rangle \\
& =\sum_{w=u v}\langle s, u\rangle \sum_{v=v_{1} v_{2} \ldots} \prod_{n \geq 1}\left\langle s_{n}, v_{n}\right\rangle \\
& =\sum_{w=u v_{1} v_{2} \ldots}\langle s, u\rangle \prod_{n \geq 1}\left\langle s_{n}, v_{n}\right\rangle \\
& =\sum_{w=v_{1} v_{2} \ldots}\left\langle s_{n}^{\prime}, v_{n}\right\rangle \\
& =\left\langle\prod_{n \geq 1} s_{n}^{\prime}, w\right\rangle,
\end{aligned}
$$

where $s_{1}^{\prime}=s$ and $s_{n+1}^{\prime}=s_{n}$ for all $n \geq 1$.

Let again $s_{n} \in S\left\langle\left\langle X^{*}\right\rangle\right.$ for all $n \geq 1$. Suppose that the sequence $i_{1}=1 \leq i_{2} \leq \cdots$ increases without a bound. For each $n \geq 1$, define $s_{n}^{\prime}=s_{i_{n}} \cdots s_{i_{n+1}-1}$. Then for all $w \in X^{\omega}$,

$$
\begin{aligned}
\left\langle\prod_{n \geq 1} s_{n}, w\right\rangle & =\sum_{w=v_{1} v_{2} \ldots} \prod_{n \geq 1}\left\langle s_{n}, v_{n}\right\rangle \\
& =\sum_{w=v_{1} v_{2} \ldots} \prod_{n \geq 1}\left\langle s_{i_{n}}, v_{i_{n}}\right\rangle\left\langle s_{i_{n+1}-1}, v_{i_{n+1}-1}\right\rangle \\
& =\sum_{w=u_{1} u_{2} \cdots} \prod_{n \geq 1}\left\langle s_{n}^{\prime}, u_{n}\right\rangle \\
& =\left\langle\prod_{n \geq 1} s_{n}^{\prime}, w\right\rangle,
\end{aligned}
$$

proving Ax2.

Next, suppose that $s_{n}, s_{n}^{\prime} \in S\left\langle\left\langle X^{*}\right\rangle\right.$ for all $n \geq 1$. Then for all $w \in X^{\omega}$,

$$
\begin{aligned}
\left\langle\prod_{n \geq 1}\left(s_{n}+s_{n}^{\prime}\right), w\right\rangle & =\sum_{w=v_{1} v_{2} \ldots} \prod_{n \geq 1}\left\langle s_{n}+s_{n}^{\prime}, v_{n}\right\rangle \\
& =\sum_{w=v_{1} v_{2} \cdots r_{n}=s_{n}} \sum_{\text {or } r_{n}=s_{n}^{\prime}} \prod_{n \geq 1}\left\langle r_{n}, v_{n}\right\rangle \\
& =\sum_{r_{n}=s_{n} \text { or } r_{n}=s_{n}^{\prime}}\left\langle\prod_{n \geq 1} r_{n}, w\right\rangle,
\end{aligned}
$$

proving Ax3. 
Finally, suppose that $\left(I_{n}, \leq\right)$ is a nonempty directed partially ordered set for each $n \geq 1$ and $s_{i} \in S\left\langle\left\langle X^{*}\right\rangle\right.$ for all $i \in I_{n}, n \geq 1$ such that $s_{i} \leq s_{j}$ whenever $i \leq j$ in $I_{n}$. We want to prove that

$$
\prod_{n \geq 1} \sup _{i \in I_{n}} s_{i}=\sup _{i_{1} \in I_{1}, i_{2} \in I_{2}, \ldots} \prod_{n \geq 1} s_{i_{n}}
$$

Let $w \in X^{\omega}$. Then

$$
\begin{aligned}
\left\langle\prod_{n \geq 1} \sup _{i \in I_{n}} s_{i}, w\right\rangle & =\sum_{w=v_{1} v_{2} \ldots} \prod_{n \geq 1}\left\langle\sup _{i \in I_{n}} s_{i}, v_{n}\right\rangle \\
& =\sum_{w=v_{1} v_{2} \ldots} \prod_{n \geq 1} \sup _{i \in I_{n}}\left\langle s_{i}, v_{n}\right\rangle \\
& =\sum_{w=v_{1} v_{2} \ldots} \sup _{i_{1} \in I_{1}, i_{2} \in I_{2}, \ldots} \prod\left\langle s_{i_{n}}, v_{n}\right\rangle \\
& =\sup _{i_{1} \in I_{1}, i_{2} \in I_{2}, \ldots} \sum_{w=v_{1} v_{2} \ldots n \geq 1} \prod_{i_{n}}\left\langle s_{i_{n}}, v_{n}\right\rangle \\
& =\sup _{i_{1} \in I_{1}, i_{2} \in I_{2}, \ldots}\left\langle\prod_{n \geq 1} s_{i_{n}}, w\right\rangle \\
& =\left\langle\sup _{i_{1} \in I_{1}, i_{2} \in I_{2}, \ldots} \prod_{n \geq 1} s_{i_{n}}, w\right\rangle,
\end{aligned}
$$

proving Ax4.

Hence, if $S$ is a continuous semiring, then for any set $X,\left(S\left\langle\left\langle X^{*}\right\rangle, S\left\langle\left\langle X^{\omega}\right\rangle\right\rangle\right)\right.$ has a star and an omega operation.

\section{Freeness}

Suppose now that $(S, V)$ and $\left(S^{\prime}, V^{\prime}\right)$ are continuous $\omega$-semiring-semimodule pairs. We say that a pair of functions $h=\left(h_{S}, h_{V}\right)$ with $h_{S}: S \rightarrow S^{\prime}$ and $h_{V}: V \rightarrow V^{\prime}$ is a continuous $\omega$-semiring-semimodule pair morphism if $h_{S}$ is a continuous semiring homomorphism, $h_{V}$ is a continuous monoid homomorphism, $h_{S}$ and $h_{V}$ jointly preserve the action, moreover, infinite product is preserved:

$$
h_{V}\left(\prod_{n \geq 1} x_{n}\right)=\prod_{n \geq 1} h_{S}\left(x_{n}\right)
$$

for all $x_{n} \in S, n \geq 1$. In this section we prove:

Theorem 5.1. Suppose that $S$ is a continuous $\omega$-semiring. Then for each set $X$, the continuous $\omega$-semiring-semimodule pair $\left(S\left\langle\left\langle X^{*}\right\rangle, S\left\langle\left\langle X^{\omega}\right\rangle\right\rangle\right)\right.$ has the following universal property. Let $\left(S^{\prime}, V^{\prime}\right)$ be a continuous $\omega$-semiring-semimodule pair, $h_{S}$ : $S \rightarrow S^{\prime}$ a continuous semiring morphism and $h_{X}: X \rightarrow S^{\prime}$ a function. Suppose that the elements of $h_{S}(S)$ commute with the elements of $S^{\prime}$ and the following infinite commutativity holds:

$$
\prod_{n \geq 1} s_{n} s_{n}^{\prime}=\left(\prod_{n \geq 1} s_{n}\right)\left(\prod_{n \geq 1} s_{n}^{\prime}\right)
$$

for all $s_{n} \in h_{S}(S)$ and $s_{n}^{\prime} \in S^{\prime}$. Then there is a unique continuous semiringsemimodule morphism $h^{\sharp}=\left(h_{S}^{\sharp}, h_{V}^{\sharp}\right)$ extending $h_{S}$ and $h_{X}$. 
Proof. We have already shown that $\left(S\left\langle\left\langle X^{*}\right\rangle, S\left\langle\left\langle X^{\omega}\right\rangle\right)\right.\right.$ is a continuous semiringsemimodule pair. Let us first extend $h$ to a function $X^{*} \rightarrow S$, denoted just $h$, so that it becomes a (multiplicative) monoid homomorphism. Then let $h_{S}^{\sharp}: S\left\langle\left\langle X^{*}\right\rangle \rightarrow S^{\prime}\right.$ be defined by

$$
h_{S}^{\sharp}(s)=\sum_{u \in X^{*}} h_{S}(\langle s, u\rangle) h(u) .
$$

It is known that $h_{S}^{\sharp}$ is a continuous semiring homomorphism.

Next we extend $h$ to a function $X^{\omega} \rightarrow V^{\prime}$ by defining $h(v)=\prod_{i \geq 1} h_{X}\left(x_{i}\right)$ for each $v=x_{1} x_{2} \ldots$ in $X^{\omega}$. Finally, when $s \in S\left\langle\left\langle X^{\omega}\right\rangle\right.$, let $h_{V}^{\sharp}(s)=\sum_{v \in X^{\omega}} h_{S}(\langle s, v\rangle) h(v)$.

Suppose that $s_{i} \in S\left\langle\left\langle X^{\omega}\right\rangle\right\rangle$ for all $i \in I$, where $I$ is nonempty directed partially ordered set, ordered by the relation $\leq$. Moreover, suppose that $s_{i} \leq s_{j}$ whenever $i \leq j$ in $I$ and let $s=\sup _{i \in I} s_{i}$. Then

$$
\begin{aligned}
h_{V}^{\sharp}(s) & =\sum_{v \in X^{\omega}} h_{S}(\langle s, v\rangle) h(v) \\
& =\sum_{v \in X^{\omega}} \sup _{i \in I} h_{S}\left(\left\langle s_{i}, v\right\rangle\right) h(v) \\
& =\sup _{i \in I} \sum_{v \in X^{\omega}} h_{S}\left(\left\langle s_{i}, v\right\rangle\right) h(v) \\
& =\sup _{i \in I} h_{V}^{\sharp}\left(s_{i}\right),
\end{aligned}
$$

proving that $h_{V}^{\sharp}$ is continuous. To prove that $h_{V}^{\sharp}$ preserves the sum operation, let $s_{1}, s_{2} \in S\left\langle\left\langle X^{\omega}\right\rangle\right.$. Then

$$
\begin{aligned}
h_{V}^{\sharp}\left(s_{1}+s_{2}\right) & =\sum_{v \in X^{\omega}} h_{S}\left(\left\langle s_{1}+s_{2}, v\right\rangle\right) h(v) \\
& =\sum_{v \in X^{\omega}} h_{S}\left(\left\langle s_{1}, v\right\rangle\right) h(v)+h_{S}\left(\left\langle s_{2}, v\right\rangle\right) h(v) \\
& =\sum_{v \in X^{\omega}} h_{S}\left(\left\langle s_{1}, v\right\rangle\right) h(v)+\sum_{v \in X^{\omega}} h_{S}\left(\left\langle s_{2}, v\right\rangle\right) h(v) \\
& =h_{V}^{\sharp}\left(s_{1}\right)+h_{V}^{\sharp}\left(s_{2}\right) .
\end{aligned}
$$

It is clear that $h_{V}^{\sharp}$ preserves 0 . In order to prove that $h_{S}^{\sharp}$ and $h_{V}^{\sharp}$ jointly preserve the action, let $s \in S\left\langle\left\langle X^{*}\right\rangle\right.$ and $r \in S\left\langle\left\langle X^{\omega}\right\rangle\right.$. Then

$$
\begin{aligned}
h_{V}^{\sharp}(s r) & =\sum_{v \in X^{\omega}} h_{S}(\langle s r, v\rangle) h(v) \\
& =\sum_{v \in X^{\omega}} \sum_{v=u w} h_{S}(\langle s, u\rangle) h_{S}(\langle s, w\rangle) h(u) h(w) \\
& =\sum_{v \in X^{\omega}} \sum_{v=u w} h_{S}(\langle s, u\rangle) h(u) h_{S}(\langle r, w\rangle) h(w) \\
& =\sum_{u \in X^{*}} h_{S}(\langle s, u\rangle) h(u) \sum_{w \in X^{\omega}} h_{S}(\langle r, w\rangle) h(w) \\
& =h_{S}^{\sharp}(s) h_{V}^{\sharp}(r) .
\end{aligned}
$$


Finally, we prove that $h_{V}^{\sharp}$ preserves the infinite product. To this end, let $s_{n} \in$ $S\left\langle\left\langle X^{*}\right\rangle\right.$ for all $n \geq 1$. We want to prove that $h_{V}^{\sharp}\left(\prod_{n \geq 1} s_{n}\right)=\prod_{n \geq 1} h_{S}^{\sharp}\left(s_{n}\right)$.

$$
\begin{aligned}
h_{V}^{\sharp}\left(\prod_{n \geq 1} s_{n}\right) & =\sum_{v \in X^{\omega}} h_{S}\left(\left\langle\prod_{n \geq 1} s_{n}, v\right\rangle\right) h(v) \\
& =\sum_{v \in X^{\omega}} \sum_{v=v_{1} v_{2} \ldots} \prod_{n \geq 1} h_{S}\left(\left\langle s_{n}, v_{n}\right\rangle\right) h\left(v_{n}\right) \\
& =\sum_{v \in X^{\omega}} \sum_{v=v_{1} v_{2} \ldots} \prod_{n \geq 1} h_{S}\left(\left\langle s_{n}, v_{n}\right\rangle\right) \prod_{n \geq 1} h\left(v_{n}\right) \\
& =\prod_{n \geq 1}\left(\sum_{v_{n} \in X^{*}} h_{S}\left(\left\langle s_{n}, v_{n}\right\rangle\right) h\left(v_{n}\right)\right) \\
& =\prod_{n \geq 1} h_{S}\left(s_{n}\right) .
\end{aligned}
$$

It is clear that $h_{S}$ extends $h$. Since the definitions of $h_{S}$ and $h_{V}$ were forced, they are unique.

\section{The category $\operatorname{Matr}_{(S, V)}$}

All categories $\mathcal{C}$ in the paper will have sets as objects. The composition of morphisms $f: X \rightarrow Y$ and $g: Y \rightarrow Z$ will be denoted $f \circ g$. We usually let id $_{X}$ denote the identity morphism $X \rightarrow X$.

Our categories will have finite coproducts. For a sequence $X_{1}, \ldots, X_{n}$ of objects, the coproduct $X_{1} \oplus \cdots \oplus X_{n}$ will be given by disjoint union $X_{1} \uplus \cdots \uplus X_{n}$. In particular, the empty set $\varnothing$ will serve as initial object.

Let $X_{1}, \ldots, X_{n}$ be objects. For each $i=1, \ldots, n$, the $i$ th coproduct injection $\operatorname{in}_{X_{i}}: X_{i} \rightarrow X_{1} \oplus \cdots \oplus X_{n}$ will always be determined by the embedding of $X_{i}$ into $X_{1} \uplus \cdots \uplus X_{n}$. We will let $!_{X}$ denote the unique morphism $\varnothing \rightarrow X$. Moreover, if $f_{i}: X_{i} \rightarrow X$ for $i=1, \ldots, n$, then we will let $\left\langle f_{1}, \ldots, f_{n}\right\rangle$ denote the unique morphism $f: X_{1} \oplus \cdots \oplus X_{n} \rightarrow X$ with $\operatorname{in}_{X_{i}} \circ f=f_{i}$ for all $i$. And when $f_{i}: X_{i} \rightarrow Y_{i}$, where $i \in\{1, \ldots, n\}$, then we let $f_{1} \oplus \cdots \oplus f_{n}$ denote the unique morphism $f: X_{1} \oplus \cdots \oplus X_{n} \rightarrow$ $Y_{1} \oplus \cdots \oplus Y_{n}$ with $\operatorname{in}_{X_{i}} \circ f=f_{i} \circ$ in $_{Y_{i}}$ for all $i$.

For any $X, Y$, the hom-set $\mathcal{C}(X, Y)$ of morphisms $X \rightarrow Y$ will be both a complete partial order $(\mathcal{C}, \leq)$ and a commutative monoid $\left(\mathcal{C}(X, Y),+, 0_{X, Y}\right)$ such that the zero morphism $0_{X, Y}$ is also least w.r.t. $\leq$ and the operation + is continuous in both of its arguments. Also, the operation of composition will be continuous in both arguments. Moreover, the partial order will be related to the coproduct structure so that for any $f, g: X_{1} \oplus \cdots \oplus X_{n} \rightarrow Y, f \leq g$ iff $\mathbf{i n}_{X_{i}} \circ f \leq \mathbf{n}_{X_{i}} \circ g$. It follows that when $f_{i}: X_{i} \rightarrow Y_{i}$, where $i \in\{1, \ldots, n\}$, then $f_{1} \oplus \cdots \oplus f_{n} \leq g_{1} \oplus \cdots \oplus g_{n}$ iff $f_{i} \leq g_{i}$ for all $i$.

The following identities will hold for all $f, g: X \rightarrow Y$ and $h: Y \rightarrow Z$ :

$$
\begin{aligned}
(f+g) \circ h & =f \circ h+g \circ h \\
0_{X, Y} \circ h & =0_{X, Z}
\end{aligned}
$$


Finally, our categories will be equipped with a dagger operation mapping a morphism $f: X \rightarrow X \oplus Y$ to a morphism $f^{\dagger}: X \rightarrow Y$. This operation will always be a fixed point operation, so that the following fixed point identity will hold:

$$
f^{\dagger}=f \circ\left\langle f^{\dagger}, \mathbf{i d}_{Y}\right\rangle
$$

for all $f: X \rightarrow X \oplus Y$.

Iteration categories are categories with finite coproducts and a dagger operation satisfying certain identities including the above fixed point identity, the parameter identity

$$
\left(f \circ\left(\mathbf{i d}_{X} \oplus g\right)\right)^{\dagger}=f^{\dagger} \circ g
$$

where $f: X \rightarrow X \oplus Y$ and $g: Y \rightarrow Z$, the double dagger identity

$$
f^{\dagger \dagger}=\left(f \circ\left(\left\langle\mathbf{i d}_{X}, \mathbf{i d}_{X}\right\rangle \oplus \mathbf{i d}_{Y}\right)\right)^{\dagger},
$$

where $f: X \rightarrow X \oplus X \oplus Y$, to name a few, and some other identities including the group identities that we will described later. All of our categories will be iteration categories.

Suppose now that $(S, V)$ is a continuous $\omega$-semiring-semimodule pair. Then $(S, V)$ determines a category $\operatorname{Matr}_{(S, V)}$ whose objects are all sets and a morphism $I \rightarrow J$ is an ordered pair $(A, u)$, where $A \in S^{I \times J}$ and $u \in V^{I}$. Hence a morphism $I \rightarrow I$ is an element of the semiring-semimodule pair $\left(S^{I \times I}, V^{I}\right)$.

Composition is defined as follows. Suppose that $(A, u): I \rightarrow J$ and $(B, v): J \rightarrow$ $K$. Then we define $(A, u) \circ(B, v)=(A B, u+A v): I \rightarrow K$. It is easy to check that composition is associative with the morphisms $\left(E^{I \times I}, 0^{I}\right): I \rightarrow I$ serving as identities, where $E^{I \times I}$ is the unit matrix in $S^{I \times I}$ and $0^{I}$ denotes the zero element of $V^{I}$. (For finite sets, this category is defined in [1].)

The partial order $\leq$ on a hom-set of $\operatorname{Matr}_{(S, V)}$ is defined pointwise, so that when $(A, u),(B, v): I \rightarrow J$, then $(A, u) \leq(B, v)$ iff $A_{i, j} \leq B_{i, j}$ and $u_{i} \leq v_{i}$ for all $i \in I$ and $j \in J$. Clearly, each hom-set forms a complete partial order, and it is not difficult to verify that composition is continuous.

We can also impose a commutative monoid structure on the hom-sets by defining $(A, u)+(B, v)$ pointwise, for all $(A, u),(B, v): I \rightarrow J$. Hence $(A, u)+(B, v)=(C, w)$ with $C_{i, j}=A_{i, j}+B_{i, j}$ and $w_{i}=u_{i}+v_{i}$ for all $i \in I$ and $j \in J$. The zero morphism $I \rightarrow J$ is the morphism $\left(0^{I \times J}, 0^{I}\right)$ consisting of two zero matrices. We denote it by $0_{I, J}$, or just 0 . We have

$$
\begin{aligned}
((A, u)+(B, v)) & \circ(C, w)=(A, u) \circ(C, w)+(B, v) \circ(C, w) \\
0_{I, j} \circ(C, w) & =0_{I, K}
\end{aligned}
$$

for all $(C, w): J \rightarrow K$. It is not difficult to prove that composition is continuous.

Coproduct is given by disjoint union on objects. When $X_{1}, \ldots, X_{n}$ is a sequence of sets and $i \in\{1, \ldots, n\}$, then $i$ th coproduct embedding $\operatorname{in}_{i}$ consists of an $X_{i} \times\left(X_{1} \uplus\right.$ $\left.\cdots \uplus X_{n}\right)$ matrix whose $x_{i} \times X_{i}$ submatrix is an identity matrix and whose $X_{i} \times X_{j}$ matrices are all zero matrices for $j \neq i$, together with the column matrix $0^{X_{i}} \in V^{X_{i}}$. 
We have already noted that for each set $I,\left(S^{I \times I}, V^{I}\right)$ is a continuous $\omega$-semiringsemimodule pair. Hence it comes with a star and an omega operation: For each $A \in S^{I \times I}, A^{*}=\sum_{n \geq 0} A^{n} \in S^{I \times I}$ and $A^{\omega}=\prod_{n \geq 1} A$ in $V^{I}$. These operations satisfy the identities mentioned above. And in fact,

$$
\begin{aligned}
(A+B)^{*} & =\left(A^{*} B\right)^{*} A^{*}, \quad A, B \in S^{I \times I} \\
(A B)^{*} & =E_{I}+A(B A)^{*} B, \quad A \in S^{I \times J}, B \in S^{J \times I}
\end{aligned}
$$

and

$$
\begin{aligned}
(A+B)^{\omega} & =\left(A^{*} B\right)^{*} A^{\omega}+\left(A^{*} B\right)^{\omega}, \quad A, B \in S^{I \times I} \\
(A B)^{\omega} & =A(B A)^{\omega}, \quad A \in S^{I \times J}, B \in S^{J \times I}
\end{aligned}
$$

Suppose now that $I=J \uplus K$ and $M \in S^{I \times I}$ is partitioned as

$$
M=\left(\begin{array}{ll}
a & b \\
c & d
\end{array}\right) .
$$

Then

$$
M^{*}=\left(\begin{array}{ll}
\left(a+b d^{*} c\right)^{*} & \left(a+b d^{*} c\right)^{*} b d^{*} \\
\left(d+c a^{*} b\right)^{*} c a^{*} & \left(d+c a^{*} b\right)^{*}
\end{array}\right)
$$

and

$$
M^{\omega}=\left(\begin{array}{c}
\left(a+b d^{*} c\right)^{\omega}+\left(a+b d^{*} c\right)^{*} b d^{\omega} \\
\left(d+c a^{*} b\right)^{\omega}+\left(d+c a^{*} b\right)^{*} c a^{\omega}
\end{array}\right)
$$

The star and omega operations together give rise to a dagger operation over $\mathbf{M a t r}_{(S, V)}$ that map a morphism $X \rightarrow X \oplus Y$ to a morphism $X \rightarrow Y$. To define it, let $(A, u): X \rightarrow I \oplus J$, and partition $A$ as $(a, b)$ with $a \in S^{I \times I}$ and $b \in S^{I \times J}$. Then we define $(A, u)^{\dagger}=\left(a^{*} b, a^{\omega}+a^{*} v\right): I \rightarrow J$. Clearly, $(A, u)^{\dagger}$ is a solution of the equation

$$
(X, x)=(A, u)\left(\begin{array}{c}
(X, x) \\
\left(E_{J}, 0\right)
\end{array}\right)=(a X+b, a x+u)
$$

where $(X, x)$ ranges over the morphisms $I \rightarrow J$. It is known that equipped with dagger, $\operatorname{Matr}_{(S, V)}$ is an iteration category.

\section{Categories of Series}

\subsection{The category $\operatorname{Ser}_{S, \Sigma}$}

Suppose that $S$ is a commutative continuous semiring and $\Sigma$ is a set. We define the category $\operatorname{Ser}_{S, \Sigma}$ whose objects are all sets and a morphism $X \rightarrow Y$ is a function $f: X \rightarrow S\left\langle\left\langle(Y \uplus \Sigma)^{*}\right\rangle\right.$, or alternatively, a tuple $\left(f_{x}\right)_{x \in X}$ of series $f_{x} \in S\left\langle\left\langle(Y \uplus \Sigma)^{*}\right\rangle\right.$. Suppose that $f: X \rightarrow Y$ and $g: Y \rightarrow Z$. Then we define $f \circ g$ as the function composition of $f$ and $g^{\sharp}$, the extension of the function $Y \uplus \Sigma$ to $S\left\langle\left\langle(Z \uplus \Sigma)^{*}\right\rangle\right\rangle$ which 
agrees with $g$ on $Y$ and is the identity function on $\Sigma$ to a continuous semiring homomorphism $S\left\langle(Y \uplus \Sigma)^{*}\right\rangle \rightarrow S\left\langle\left\langle(Z \uplus \Sigma)^{*}\right\rangle\right\rangle$. For each $X$, the identity morphism id $_{X}$ is the embedding of $X$ into $X \uplus \Sigma$.

Each hom-set of morphisms $X \rightarrow Y$ of the category $\mathbf{S e r}_{S, \Sigma}$ has the structure of a complete partial order and commutative monoid. For any $f, g: X \rightarrow Y$, we define $f \leq g$ iff $f_{x} \leq g_{x}$ for all $x$, and similarly, $(f+g)_{x}=f_{x}+g_{x}$ for all $x$. The neutral element is the series $0_{X, Y}$ whose components are all 0 . This is also the least morphism $X \rightarrow Y$. Composition of morphisms is continuous as is the sum operation.

Also, $\mathbf{S e r}_{S}$ has finite coproducts are given by disjoint sum on objects. The coproduct $X_{1} \oplus \cdots \oplus X_{n}$ of a sequence $X_{1}, \ldots, X_{n}$ of sets is given by disjoint union, and for each $i \in\{1, \ldots, n\}, \operatorname{in}_{X_{i}}: X_{i} \rightarrow X_{1} \oplus \cdots \oplus X_{n}$ is the embedding of $X_{i}$ into $S\left\langle\left(X_{1} \uplus \cdots \uplus X_{n} \uplus \Sigma\right)^{*}\right\rangle$.

We define a dagger operation on $\operatorname{Ser}_{S, \Sigma}$ which maps a morphism $f: X \rightarrow X \oplus Y$ to $f^{\dagger}: X \rightarrow Y$. The morphism $f^{\dagger}$ is given as the least solution of the fixed point equation

$$
\xi=f \circ\left\langle\xi, \mathbf{i d}_{Y}\right\rangle .
$$

In more detail, $f^{\dagger}=\sup _{n \geq 0} f^{(n)}$, where $f^{(0)}=0$ and $f^{(n+1)}=f \circ\left\langle f^{(n)}, \mathbf{i d}_{Y}\right\rangle$. It is known that equipped with this dagger operation, $\mathbf{S e r}_{S, \Sigma}$ is an iteration category.

\subsection{The category $\operatorname{Ser}_{S, \Sigma}^{\omega}$.}

Suppose now that $S$ is a commutative continuous $\omega$-semiring satisfying the infinite commutativity identity. Then we define another category Ser $_{S, \Sigma}^{\omega}$ with sets as objects as above. However, a morphism $f: X \rightarrow Y$ is now a triplet $\left(f_{0}, f_{M}, f_{\omega}\right)$ with $f_{0}: X \rightarrow Y$ in $\operatorname{Ser}_{S, \Sigma}$ and $\left(f_{M}, f_{\omega}\right): X \rightarrow Y$ in $\operatorname{Matr}_{\left(S\left\langle(Y \uplus \Sigma)^{*}\right\rangle, S\left\langle(Y \uplus \Sigma)^{\omega}\right\rangle\right)}$. Hence, $\left.f_{0}: X \rightarrow S\left\langle(Y \uplus \Sigma)^{*}\right\rangle, f_{M} \in S\left\langle(Y \uplus \Sigma)^{*}\right\rangle\right\rangle^{X \times Y}$ and $\left.f_{\omega}: X \rightarrow S\left\langle(Y \uplus \Sigma)^{\omega}\right\rangle\right\rangle$.

Composition is defined as follows. Let $f: X \rightarrow Y$ and $g: Y \rightarrow Z$. Then the components of $h=f \circ g: X \rightarrow Z$ are given by

- $h_{0}=f_{0} \circ g_{0}$, where the composition is taken from $\mathbf{S e r}_{S, \Sigma}$, and

- $\left(h_{M}, h_{\omega}\right)=g_{0}^{\sharp}\left(\left(f_{M}, f_{\omega}\right)\right) \circ\left(g_{M}, g_{\omega}\right)=\left(g_{0}^{\sharp}\left(f_{M}\right), g_{0}^{\sharp}\left(f_{\omega}\right)\right) \circ\left(g_{M}, g_{\omega}\right)$ where the composition is taken from the category $\operatorname{Matr}_{\left(S\left\langle(Z \uplus \Sigma)^{*}\right\rangle, S\left\langle(Z \uplus \Sigma)^{\omega}\right\rangle\right) \text {. }}$.

Note that the definition is legitimate, since $\left(S\left\langle(Z \uplus \Sigma)^{*}\right\rangle, S\left\langle\left\langle(Z \uplus \Sigma)^{\omega}\right\rangle\right)\right.$ is a continuous $\omega$-semiring-semimodule pair and $g_{0}^{\sharp}\left(\left(f_{M}, f_{\omega}\right)\right)=\left(g_{0}^{\sharp}\left(f_{M}\right), g_{0}^{\sharp}\left(f_{\omega}\right)\right)$ is a mor-

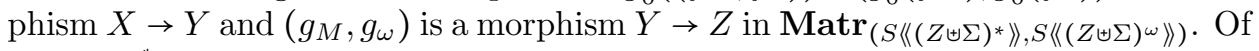
course, $g_{0}^{\sharp}$ is the extension of $g_{0}$ to a continuous $\omega$-semiring-semimodule morphism and $g_{0}^{\sharp}\left(f_{M}\right)$ and $g_{0}^{\sharp}\left(f_{\omega}\right)$ are formed component-wise. It is a routine matter to verify that composition is associative. The identity morphism $X \rightarrow X$ is determined by

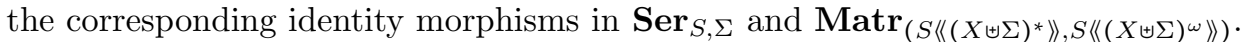

Each hom-set of morphisms $X \rightarrow Y$ is partially ordered by the component-wise

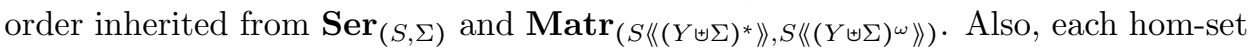


has the structure of a commutative monoid. For any morphisms $f=\left(f_{0}, f_{M}, f_{\omega}\right)$ and $g=\left(g_{0}, g_{M}, g_{\omega}\right): X \rightarrow Y$, we define

$$
f+g=\left(f_{0}+g_{0}, f_{M}+g_{M}, f_{\omega}+g_{\omega}\right) .
$$

The components of the morphism $0_{X, Y}$ are the respective zero morphisms.

The category $\operatorname{Ser}_{S, \Sigma}^{\omega}$ has finite coproducts. On objects, coproduct is again given by disjoint sum.

We now define a dagger operation. Let $f: X \rightarrow X \oplus Y, f=\left(f_{0}, f_{M}, f_{\omega}\right)$. Then we define the components of $f^{\dagger}: X \rightarrow Y$ as $f_{0}^{\dagger}: X \rightarrow Y$ in $\operatorname{Ser}_{S, \Sigma}$ and

$$
\left(\left(f^{\dagger}\right)_{M},\left(f^{\dagger}\right)_{\omega}\right)=\left\langle f_{0}^{\dagger}, \mathbf{i d}_{Y}\right\rangle^{\sharp}\left(\left(f_{M}, f_{\omega}\right)^{\dagger}\right)=\left(\left\langle f_{0}, \mathbf{i d}_{Y}\right\rangle^{\sharp}\left(f_{M}, f_{\omega}\right)^{\dagger}\right): X \rightarrow Y
$$

in $\operatorname{Matr}_{\left(S\left\langle(Y \uplus \Sigma)^{*}\right\rangle, S\left\langle(Y \uplus \Sigma)^{\omega}\right\rangle\right)}$.

We prove that the fixed point identity holds. To this end, let $f: X \rightarrow X \oplus Y$ as above. Then

$$
f \circ\left\langle f^{\dagger}, \mathbf{i d}_{Y}\right\rangle=\left(\left\langlef_{0} \circ\left\langle f_{0}^{\dagger}, \mathbf{i d}_{Y}\right\rangle,\left\langle f_{0}^{\dagger}, \mathbf{i d}_{Y}\right\rangle^{\sharp}\left(\left(f_{M}, f_{\omega}\right)\right) \circ\left\langle\left\langle f_{0}^{\dagger}, \mathbf{i d}_{Y}\right\rangle^{\sharp}\left(\left(f_{M}, f_{\omega}\right)^{\dagger}\right), \mathbf{i d}_{Y}\right\rangle .\right.\right.
$$

Hence, the first component of $f^{\dagger}$ is $f_{0}^{\dagger}$. The second and third are given by

$$
\begin{aligned}
\left\langle f_{0}^{\dagger}, \mathbf{i d}_{Y}\right\rangle^{\sharp} & \left.\left(\left(f_{M}, f_{\omega}\right)\right) \circ\left\langle\left\langle f_{0}^{\dagger}, \mathbf{i d}_{Y}\right\rangle^{\sharp}\left(\left(f_{M}, f_{\omega}\right)^{\dagger}\right)\right), \mathbf{i d}_{Y}\right\rangle \\
& \left.=\left\langle f_{0}^{\dagger}, \mathbf{i d}_{Y}\right\rangle^{\sharp}\left(\left(f_{M}, f_{\omega}\right) \circ\left\langle\left(f_{M}, f_{\omega}\right)^{\dagger}\right), \mathbf{i d}_{Y}\right\rangle\right) \\
& =\left\langle f_{0}^{\dagger}, \mathbf{i d}_{Y}\right\rangle^{\sharp}\left(\left(f_{M}, f_{\omega}\right)^{\dagger}\right) .
\end{aligned}
$$

Hence $f \circ\left\langle f^{\dagger}, \mathbf{i d}_{Y}\right\rangle=f^{\dagger}$.

Theorem 7.1. $\operatorname{Ser}_{(S, \Sigma)}^{\omega}$ is an iteration category.

Proof. We have already proved that the fixed point identity holds. In order to complete the proof, we establish the parameter, double dagger and group identities.

First we consider the parameter identity. Let $f=\left(f_{0}, f_{M}, f_{\omega}\right): X \rightarrow X \oplus Y$ and $g=\left(g_{0}, g_{M}, g_{\omega}\right): Y \rightarrow Z$. We want to prove that $\left(f \circ\left(\mathbf{i d}_{X} \oplus g\right)\right)^{\dagger}=f^{\dagger} \circ g$.

It is clear that the first components of $\left(f \circ\left(\mathbf{i d}_{X} \oplus g\right)\right)^{\dagger}$ and $f^{\dagger} \circ g$ are $\left(f_{0} \circ\right.$ $\left.\left(\mathbf{i d}_{X} \oplus g_{0}\right)\right)^{\dagger}$ and $f_{0}^{\dagger} \circ g$, respectively. Since the parameter identity holds in $\mathbf{S e r}_{S, \Sigma}$, we conclude that the first components are equal.

The second and third components of $f \circ\left(\mathbf{i d}_{X} \oplus g\right)$ are given by

$$
\left(\mathbf{i d}_{X} \oplus g_{0}\right)^{\sharp}\left(\left(f_{M}, f_{\omega}\right)\right) \circ\left(\mathbf{i d}_{X} \oplus\left(g_{M}, g_{\omega}\right)\right),
$$

hence the corresponding components of $\left(f \circ\left(\mathbf{i d}_{X} \oplus g\right)\right)^{\dagger}$ are given by

$$
\left\langle\left(f_{0} \circ\left(\mathbf{i d}_{X} \oplus g_{0}\right)\right)^{\dagger}, \mathbf{i d}_{Z}\right\rangle^{\sharp}\left(\left(\left(\mathbf{i d}_{X} \oplus g_{0}\right)^{\sharp}\left(\left(f_{M}, f_{\omega}\right)\right) \circ\left(\mathbf{i d}_{X} \oplus\left(g_{M}, g_{\omega}\right)\right)\right)^{\dagger}\right)
$$

which is

$$
\begin{aligned}
& \left\langle f_{0}^{\dagger} \circ g_{0}, \mathbf{i d}_{Z}\right\rangle^{\sharp}\left(\left(\left(\mathbf{i d}_{X} \oplus g_{0}\right)^{\sharp}\left(\left(f_{M}, f_{\omega}\right)\right)\right)^{\dagger} \circ\left(g_{M}, g_{\omega}\right)\right) \\
& =\left(\left(\mathbf{i d}_{X} \oplus g_{0}\right) \circ\left\langle f_{0}^{\dagger} \circ g_{0}, \mathbf{i d}_{Z}\right\rangle\right)^{\sharp}\left(\left(f_{M}, f_{\omega}\right)^{\dagger}\right) \circ\left\langle f_{0}^{\dagger} \circ\left\langle g_{0}, \mathbf{i d}_{Z}\right\rangle^{\sharp}\left(g_{M}, g_{\omega}\right)\right. \\
& =\left(\left(\mathbf{i d}_{X} \oplus g_{0}\right) \circ\left\langle f_{0}^{\dagger} \circ g_{0}, \mathbf{i d}_{Z}\right\rangle\right)^{\sharp}\left(\left(f_{M}, f_{\omega}\right)^{\dagger}\right) \circ\left(g_{M}, g_{\omega}\right) .
\end{aligned}
$$


On the other hand, the second and third components of $f^{\dagger}$ and $f^{\dagger} \circ g$ are respectively given by $\left\langle f_{0}^{\dagger}, \mathbf{i d}_{Y}\right\rangle^{\sharp}\left(\left(f_{M}, f_{\omega}\right)^{\dagger}\right)$ and

$$
g_{0}^{\sharp}\left(\left\langle f_{0}^{\dagger}, \mathbf{i d}_{Y}\right\rangle^{\sharp}\left(\left(f_{M}, f_{\omega}\right)^{\dagger}\right)\right) \circ\left(g_{M}, g_{\omega}\right)=\left(\left\langle f_{0}^{\dagger}, \mathbf{i d}_{Y}\right\rangle \circ g_{0}\right)^{\sharp}\left(\left(f_{M}, f_{\omega}\right)^{\dagger}\right) \circ\left(g_{M}, g_{\omega}\right) .
$$

But $\left(\mathbf{i d}_{X} \oplus g_{0}\right) \circ\left\langle f_{0}^{\dagger} \circ g_{0}, \mathbf{i d}_{Z}\right\rangle=\left\langle f_{0}^{\dagger} \circ g_{0}, g_{0}\right\rangle=\left\langle f_{0}^{\dagger}, \mathbf{i d}_{Y}\right\rangle \circ g_{0}$, so that the second and third components are also equal.

We prove that the double dagger identity holds. To this end, let $f=\left(f_{0}, f_{M}, f_{\omega}\right)$ : $X \rightarrow X \oplus X \oplus Y$ and $\tau=\left\langle\mathbf{i d}_{X}, \mathbf{i d}_{X}\right\rangle \oplus \mathbf{i d}_{Y}$. Then the first component of $f^{\dagger \dagger}$ is $f_{0}^{\dagger \dagger}$, and the first component of $(f \circ \tau)^{\dagger}$ is $\left(f_{0} \circ \tau\right)^{\dagger}$. These are equal since the double dagger identity holds in $\mathbf{S e r}_{S, \Sigma}$.

The second and third components of $f^{\dagger}$ and $f^{\dagger \dagger}$ are

$$
\left\langle f_{0}^{\dagger}, \mathbf{i d}_{X \oplus Y}\right\rangle^{\sharp}\left(\left(f_{M}, f_{\omega}\right)^{\dagger}\right)
$$

and

$$
\begin{aligned}
& \left.\left\langle f_{0}^{\dagger \dagger}, \mathbf{i d}_{Y}\right\rangle^{\sharp}\left(\left\langle f_{0}^{\dagger}, \mathbf{i} \mathbf{d}_{X \oplus Y}\right\rangle^{\sharp}\left(\left(f_{M}, f_{\omega}\right)^{\dagger}\right)\right)\right\rangle \\
& =\left(\left\langle f_{0}^{\dagger}, \mathbf{i d}_{X \oplus Y}\right\rangle \circ\left\langle f_{0}^{\dagger \dagger}, \mathbf{i d}_{Y}\right\rangle\right)^{\sharp}\left(\left(f_{M}, f_{\omega}\right)^{\dagger \dagger}\right) \\
& =\left(\left\langle f_{0}^{\dagger} \circ\left\langle f_{0}^{\dagger \dagger}, \mathbf{i d}_{Y}\right\rangle, f_{0}^{\dagger \dagger}, \mathbf{i d}_{Y}\right)^{\sharp}\left(\left(f_{M}, f_{\omega}\right)^{\dagger \dagger}\right)\right. \\
& =\left\langle f_{0}^{\dagger \dagger}, f_{0}^{\dagger \dagger}, \mathbf{i d}_{Y}\right\rangle^{\sharp}\left(\left(f_{M}, f_{\omega}\right)^{\dagger \dagger}\right) \\
& =\left(\tau \circ\left\langle f^{\dagger \dagger}, \mathbf{i d}_{Y}\right\rangle\right)^{\sharp}\left(\left(f_{M}, f_{\omega}\right)^{\dagger \dagger}\right),
\end{aligned}
$$

respectively. Now the second and third components of $f \circ \tau$ are given by $\tau^{\sharp}\left(\left(f_{M}, f_{\omega}\right)\right) \circ$ $\tau$ and thus the second and third components of $(f \circ \tau)^{\dagger}$ are given by

$$
\begin{aligned}
& \left\langle(f \circ \tau)^{\dagger}, \mathbf{i d}_{Y}\right\rangle^{\sharp}\left(\left(\tau^{\sharp}\left(\left(f_{M}, f_{\omega}\right)\right) \circ \tau\right)^{\dagger}\right) \\
& =\left\langle f^{\dagger \dagger}, \mathbf{i d}_{Y}\right\rangle^{\sharp}\left(\left(\tau^{\sharp}\left(\left(f_{M}, f_{\omega}\right)\right) \circ \tau\right)^{\dagger}\right) \\
& =\left(\left\langle f^{\dagger \dagger}, \mathbf{i d}_{Y}\right\rangle\right)^{\sharp}\left(\left(\tau^{\sharp}\left(\left(f_{M}, f_{\omega}\right)\right)\right)^{\dagger \dagger}\right) \\
& =\left(\left\langle f^{\dagger \dagger}, \mathbf{i d}_{Y}\right\rangle\right)^{\sharp}\left(\tau^{\sharp}\left(\left(\left(f_{M}, f_{\omega}\right)\right)^{\dagger \dagger}\right)\right) \\
& =\left(\tau \circ\left\langle f^{\dagger \dagger}, \mathbf{i d}_{Y}\right\rangle\right)^{\sharp}\left(\left(f_{M}, f_{\omega}\right)^{\dagger \dagger}\right) .
\end{aligned}
$$

Hence the double dagger identity holds.

Our next task is to prove that the (simplified) composition identity holds:

$$
(f \circ g)^{\dagger}=f \circ\left(g \circ\left(f \oplus \mathbf{i d}_{Z}\right)\right)^{\dagger},
$$

where $f: X \rightarrow Y$ and $g: Y \rightarrow X \oplus Z$. We will establish this identity only in the case when $Z$ is the initial object $\varnothing$. In this case, the identity takes the following form:

$$
(f \circ g)^{\dagger}=f \circ(g \circ f)^{\dagger},
$$

where $f: X \rightarrow Y$ and $g: Y \rightarrow X$. 
It is again clear that the first components of the two sides are equal, since the identity holds in $\mathbf{S e r}_{S, \Sigma}$.

Now the second and third components of $(f \circ g)^{\dagger}$ are

$$
\begin{aligned}
& \left(\left(f_{0} \circ g_{0}\right)^{\dagger}\right)^{\sharp}\left(\left(g_{0}^{\sharp}\left(\left(f_{M}, f_{\omega}\right)\right) \circ\left(g_{M}, g_{\omega}\right)\right)^{\dagger}\right) \\
& \left.=\left(\left(g_{0} \circ\left(f_{0} \circ g_{0}\right)^{\dagger}\right)^{\sharp}\left(\left(f_{M}, f_{\omega}\right)\right) \circ\left(f_{0} \circ g_{0}\right)^{\dagger}\right)^{\sharp}\left(\left(g_{M}, g_{\omega}\right)\right)\right)^{\dagger} \\
& =\left(\left(\left(g_{0} \circ f_{0}\right)^{\dagger}\right)^{\sharp}\left(\left(f_{M}, f_{\omega}\right)\right) \circ\left(\left(f_{0} \circ g_{0}\right)^{\dagger}\right)^{\sharp}\left(\left(g_{M}, g_{\omega}\right)\right)\right)^{\dagger} .
\end{aligned}
$$

The corresponding components of $f \circ(g \circ f)^{\dagger}$ are

$$
\begin{aligned}
& \left(\left(g_{0} \circ f_{0}\right)^{\dagger}\right)^{\sharp}\left(\left(f_{M}, f_{\omega}\right)\right) \circ\left(\left(g_{0} \circ f_{0}\right)^{\dagger}\right)^{\sharp}\left(\left(f_{0}^{\sharp}\left(\left(g_{M}, g_{\omega}\right)\right) \circ\left(f_{M}, f_{\omega}\right)\right)^{\dagger}\right) \\
& =\left(\left(g_{0} \circ f_{0}\right)^{\dagger}\right)^{\sharp}\left(\left(f_{M}, f_{\omega}\right)\right) \circ\left(\left(\left(f_{0} \circ g_{0}\right)^{\dagger}\right)^{\sharp}\left(\left(g_{M}, g_{\omega}\right)\right) \circ\left(\left(g_{0} \circ f_{0}\right)^{\dagger}\right)^{\sharp}\left(\left(f_{M}, f_{\omega}\right)\right)\right)^{\dagger} \\
& =\left(\left(\left(g_{0} \circ f_{0}\right)^{\dagger}\right)^{\sharp}\left(\left(f_{M}, f_{\omega}\right)\right) \circ\left(\left(f_{0} \circ g_{0}\right)^{\dagger}\right)^{\sharp}\left(\left(g_{M}, g_{\omega}\right)\right)\right)^{\dagger} .
\end{aligned}
$$

Hence, the second and third components are also equal.

Our last task is to prove that the group identities hold. Suppose that $G$ is a finite group of order $n$ whose elements are the integers $\{1, \ldots, n\}$, say. Let $g$ : $X \rightarrow X \oplus \cdots \oplus X \oplus Y$, where there are $n$ occurrences of $X$ in the target. For each $i \in\{1, \ldots, n\}$, let

$$
\rho_{i}=\left\langle\mathbf{i n}_{i \cdot 1}, \ldots, \mathbf{i n}_{i \cdot n}\right\rangle \oplus \mathbf{i d}_{Y}: X \oplus \cdots \oplus X \oplus Y \rightarrow X \oplus \cdots \oplus X \oplus Y
$$

Moreover, let

$$
\tau=\left\langle\mathbf{i d}_{X}, \ldots, \mathbf{i d}_{X}\right\rangle: X \oplus \cdots \oplus X \rightarrow X .
$$

The group identity associated with $G$ is

$$
\left\langle g \circ \rho_{1}, \ldots, g \circ \rho_{n}\right\rangle^{\dagger}=\tau \circ\left(g \circ\left(\tau \oplus \mathbf{i d}_{Y}\right)\right)^{\dagger} .
$$

We will prove this only in the case when $Y=\varnothing$. The first components of the two sides are again equal. The second and third components of the morphism on the left hand side are given by

$$
\begin{aligned}
& \left(\left\langle g_{0} \circ \rho_{1}, \ldots, g_{0} \circ \rho_{n}\right\rangle^{\dagger}\right)^{\sharp}\left(\left\langle\left(g_{M}, g_{\omega}\right) \circ \rho_{1}, \ldots,\left(g_{M}, g_{\omega}\right) \circ \rho_{n}\right\rangle^{\dagger}\right) \\
& =\left(\tau \circ\left(g_{0} \circ \tau\right)^{\dagger}\right)^{\sharp}\left(\left\langle\left(g_{M}, g_{\omega}\right) \circ \rho_{1}, \ldots,\left(g_{M}, g_{\omega}\right) \circ \rho_{n}\right\rangle^{\dagger}\right) \\
& =\left\langle\left(\tau \circ\left(g_{0} \circ \tau\right)^{\dagger}\right)^{\sharp}\left(\left(g_{M}, g_{\omega}\right)\right) \circ \rho_{1}, \ldots,\left(\tau \circ\left(g_{0} \circ \tau\right)^{\dagger}\right)^{\sharp}\left(\left(g_{M}, g_{\omega}\right)\right) \circ \rho_{n}\right\rangle^{\dagger} \\
& =\tau \circ\left(\left(\tau \circ\left(g_{0} \circ \tau\right)^{\dagger}\right)^{\sharp}\left(\left(g_{M}, g_{\omega}\right)\right) \circ \tau\right)^{\dagger} .
\end{aligned}
$$

But this is exactly the morphism determined by the second and third components of the morphism on the right hand side of the group identity associated with $G$. This completes the proof of the theorem. 


\section{Remark of the second author.}

From May 17 to May 20, 2016, Zoltan stayed and worked in my home and almost finished his presumably final paper as coauthor. He wrote this paper in his typical style of working: After extensive discussions he typed the tex file directly into the computer without using a concept or notes. This paper is complete except for the announced "Conclusion". His planned content of the "Conclusion" is unknown to me and I have not tried to reconstruct it. Therefore, this paper appears according to Zoltan's conceptions.

\section{References}

[1] S.L. Bloom and Z. Ésik: Iteration Theories. Springer, 1993.

[2] S.L. Bloom, Z. Ésik: Axiomatizing rational power series over natural numbers. Inf. Comput. 207(2009), 793-811.

[3] J.H. Conway: Regular Algebra and Finite Machines, Chapman and Hall, Ltd., 1971.

[4] Z. Ésik: Group axioms for iteration, Information and Computation, 148(1999), $131-180$.

[5] Z. Ésik: Equational properties of fixed point operations in cartesian categories: An overview. In: MFCS 2015, Springer, LNCS 9234, 2015, 18-37.

[6] Z. Ésik and W. Kuich, Modern Automata Theory, available from http://www.dmg.tuwien.ac.at/kuich/

[7] Z. Ésik and W. Kuich: Solving fixed point equations over complete semirings, to appear in Festschrift for Janusz Brzozowski's 80th birthday, World Scientific, 2017.

[8] J. Golan: Semirings and their Applications, Springer, 1999.

[9] J. Golan: Semirings and Affine Equations over Them: Theory and Applications, Springer, 2003.

[10] R.S. Cohen, A.Y. Gold: Theory of omega-Languages. I. Characterizations of omega-Context-Free Languages. J. Comput. Syst. Sci., 15(1977), 169-184.

[11] W. Kuich and A. Salomaa: Semirings, Automata, Languages, Springer, 1986.

[12] G. Markowsky: Chain complete posets and directed sets with applications, Algebra Universalis, 6(1976), 53-68. 